\title{
INTERFACES DE VISUALIZAÇÃO DE TRILHAS DE APRENDIZAGEM: UMA ANÁLISE A PARTIR DOS PRINCÍPIOS DOS SISTEMAS DE HIPERTEXTO
}

Resumo: As trilhas de aprendizagem podem ser consideradas um conjunto sistemático e multimodal de unidades de aprendizagem, contendo diferentes esquemas de navegação, que podem ir desde modelos lineares, prescritivos, passando-se por modelos mais hierárquicos, em que são permitidas escolhas, e chegando-se a modelos em rede, cuja navegação é mais livre e tem como propósito o desenvolvimento de competências. Esses esquemas de navegação podem ser personalizados, com base em variáveis, como: objetivos, perfil do aluno e características de aprendizagem. Este artigo tem como objetivo apresentar os resultados de uma das etapas de pesquisa de mestrado, que teve como um dos objetivos esboçar um metamodelo conceitual de representação dessas trilhas de aprendizagem. Para alcance desse objetivo, foi conduzida análise de dados por meio das etapas sugeridas no método da análise de conteúdo. Os resultados indicaram que, por meio da metodologia adotada, foi possível identificar uma variedade de categorias e entidades que foram usadas na composição do modelo conceitual para organização e representação de trilhas de aprendizagem. Entretanto, observou-se necessidade de refinamento dos elementos identificados, principalmente do ponto de vista de tratamento terminológico, o que evidencia a importância do uso de um vocabulário controlado, visando o enriquecimento semântico.

Palavras-chave: Trilhas de Aprendizagem. Representação da Informação. Hipertexto.
Patrícia Lopes Lopes

Doutoranda em Ciência da Informação pela Universidade

Federal de Minas Gerais

(UFMG).

patricialopesvirtual@gmail.com

Gercina Angela Lima

Pós-doutora em Estudos sobre metodologias para o processo de indexação de documentos textuais, pela Universidad Carlos

III, Madrid, Espanha, e pela

Universidade de São Paulo (USP). limagercina@gmail.com

\section{INTERFACES OF VISUALIZATION OF LEARNING TRAILS: AN ANALYSIS FROM THE PRINCIPLES OF HYPERTEXT SYSTEMS}

\begin{abstract}
The learning paths can be considered a systematic and multimodal set of learning units, containing different navigation schemes, which can range from linear, prescriptive models, passing through more hierarchical models, in which choices are allowed, and arriving to network models, whose navigation is freer and aimed at developing skills. These navigation schemes can be customized, based on variables such as: objectives, student profile and learning characteristics. This article aims to present the results of one of the Master's research stages, which had as one of the objectives to outline a conceptual metamodel of representation of these learning paths. To achieve this goal, data analysis was conducted through the steps suggested in the content analysis method. The results indicated that, through the adopted methodology, it was possible to identify a variety of categories and entities that were used in the composition of the conceptual model for organization and representation of learning paths. However, there was a need to refine the identified elements, mainly from the point of view of terminological treatment, which highlights the importance of using a controlled vocabulary, aiming at semantic enrichment.
\end{abstract}

Keywords: Learning Paths. Representation of Information. Hypertext. 


\section{INTRODUÇÃO}

Este artigo apresenta um extrato dos resultados de uma pesquisa de mestrado realizada no âmbito do Programa de Pós-Graduação em Gestão e Organização do Conhecimento da Escola de Ciência da Informação, Universidade Federal de Minas Gerais, Brasil, intitulada Proposta de metodologia para organização e representação de trilhas de aprendizagem no contexto da Educação Corporativa na Administração Pública (LOPES, 2018).

Essa pesquisa teve como objetivo geral elaborar uma metodologia para organização e representação de trilhas, com base nos princípios do hipertexto, e como objetivos específicos: (1) evidenciar de que modo as estratégias para organização e representação de trilhas de aprendizagem identificadas na revisão de literatura estavam aderentes aos princípios de estruturação de sistemas de hipertexto; (2) identificar elementos comuns de estratégias de organização e representação de trilhas aplicados de forma empírica ou referenciada; e (3) esboçar um metamodelo conceitual de representação dessas trilhas.

Os resultados apresentados neste artigo focam, especificamente, no terceiro objetivo específico proposto. Com o intuito de criar o supracitado metamodelo conceitual de representação de trilhas de aprendizagem, foram analisadas interfaces de trilhas localizadas durante a revisão de literatura. A partir dessa análise, foi possível identificar uma variedade de categorias e entidades que foram usadas para composição de um modelo conceitual para organização e representação de trilhas de aprendizagem. Mesmo em face da necessidade de refinamento dos elementos identificados, principalmente do ponto de vista de tratamento terminológico, considerou-se que o levantamento realizado contemplou necessidades reais de usuários finais.

\section{REFERENCIAL TEÓRICO}

Entende-se como trilhas de aprendizagem um conjunto sistemático e multimodal de unidades de aprendizagem, contendo diferentes esquemas de navegação, que podem ir desde modelos lineares, prescritivos, passando-se por modelos mais hierárquicos, em que são permitidas escolhas, e chegando-se a modelos em rede, cuja navegação é mais livre e tem como propósito o desenvolvimento de competências. Esses esquemas de navegação podem ser 
personalizados, com base em variáveis, como: objetivos, perfil do aluno e características de aprendizagem.

O conceito proposto envolve questões consideradas críticas nos processos de organização e representação de trilhas de aprendizagem. Com relação aos esquemas de navegação das trilhas, destacam-se os riscos e problemas adjacentes da abordagem de ensino chamado de tamanho único (ADESINA; MOLLOY, 2011; FREITAS; BRANDÃO, 2005; LUBCHAK; KUPENKO; KUZIKOV, 2012; RIVAS, 2012; GÁMEZ SUAZO; GARITA RODRÍGUEZ; CHACÓN RIVAS, 2012). O risco ou problema mencionado por esses autores representa um neologismo referente à ideia de haver uma mesma trilha de aprendizagem para alunos com diferentes conhecimentos prévios, estilos no ato de aprender, objetivos nesse ato, refletindo uma natureza prescritiva e estática. Entende-se que esse formato tamanho único se relaciona mais aos esquemas de navegação lineares, sem quaisquer níveis de customização ou personalização.

As trilhas de aprendizagem podem ser analisadas de maneira análoga aos sistemas de hipertexto. Pode-se afirmar que há aproximação entre os sistemas de hipertexto e as trilhas de aprendizagem, principalmente, quanto à arquitetura, uma vez que tanto os sistemas de hipertexto quanto as trilhas de aprendizagem possuem um esquema de representação conceitual, um conjunto de nós e links, esquemas de navegação, sistemas de armazenamento e gerenciamento de dados, interface para acesso do usuário e um sistema de autoria.

O hipertexto pode ser entendido como um esquema de organização de conteúdo textual e/ou pictórico, representado por meio de uma rede associativa de nós e links, que estabelece conexões entre ideias, conceitos e/ou itens, de forma análoga à cognição humana e que permite ao leitor ter acesso não linear à informação (LIMA, 2004, 2006, 2015; NELSON, 1965 apud BALASUBRAMANIAN, 1994; PEREZ, 1994; RADA, 1991).

Uma característica dominante do hipertexto é a abstração da rede de documentos, que permite a autores e leitores entenderem-se com mais facilidade, pois o modelo que o autor deseja comunicar poderia ser melhor expresso em hipertexto do que em um texto convencional, e o leitor poderia percorrer a rede de conceitos e seguir relacionamentos, percurso esse que seria mais difícil em um texto em papel ou linear. Essa noção de redes de conceitos relacionados aplica-se tanto a um documento, tendo um único leitor, quanto a documentos diversos, com diferentes leitores. Nesse caso, além da noção de rede de conceitos, torna-se também relevante 
a analogia do hipertexto a um grande banco de dados de documentos que pode ser apoiado pelo trabalho colaborativo (RADA, 1991).

O hipertexto pressupõe basicamente a organização de um grande corpo de informações em numerosos fragmentos, a existência de relacionamentos entre esses fragmentos e a necessidade de acesso do usuário a uma pequena fração de cada vez (SHNEIDERMAN, 1989 apud LIMA, 2015).

Do ponto de vista da arquitetura, o hipertexto pode integrar diferentes camadas (BALASUBRAMANIAN，1994; CONKLIN，1987; LEGGETT; SCHNASE; KACMAR, 1989; PEREZ, 1994), que são:

- representação conceitual ou esquema de representação: uma espécie de rede semântica que integra as informações com operações e processos. Diz respeito a uma representação conceitual para estruturar associações entre elementos de informação;

- nodos ou nós (elementos de informação): palavras, pensamentos, imagens, sons, conhecimento, fatos, dados, espaço, tempo, processos etc.;

- links (vínculos): que conectam um nodo a outro;

- esquema de navegação: estabelece um esquema de conexões (links) entre os nodos ou nós (elementos de informação);

- sistema de armazenamento e gerenciamento de dados: associa os nodos de informação da interface aos dados armazenados no banco de dados. Além disso, contempla mecanismos de recuperação da informação;

- interface para acesso do usuário: auxilia o usuário a navegar por meio de uma grande quantidade de informações, mediante ícones e links, com a ajuda de navegadores e diagramas;

- sistema de autoria: permite ao usuário criar e gerenciar nós e links.

No contexto dos sistemas de hipertexto, a interface é uma estratégia de representação do modelo organizacional da informação, que permite a visualização do conteúdo por parte de um usuário que pode mover-se de um nó a outro por meio dos links (LIMA, 2015). Especificamente sobre essa camada da arquitetura, considerando que o hipertexto pode ter diferentes tipos e ser estabelecido mediante variados esquemas conceituais, tem-se como premissa a necessidade de haver uma diversidade de interfaces. 
Apesar dessa premissa, observa-se carência de estratégias de representação de hipertextos que adotem como princípio a representação gráfica (CAMPOS, 2004). Essa lacuna também pode ser observada nas estratégias de representação de trilhas de aprendizagem, cuja maioria dos estudos (LOPES; LIMA, 2019) aponta que, apesar de haver propostas para organização do conhecimento, ou seja, abstrações que visam apresentar um modelo abstrato de trilhas, há poucas soluções de visualização desses recursos do ponto de vista do aprendiz. Muitos estudos restringem-se ao mapa conceitual, que possui limitações relacionadas à representação gráfica, principalmente em trilhas de maior complexidade ou volume de unidades de aprendizagem.

Alguns estudos apontam que há poucas interfaces para visualização de trilhas de aprendizagem (LOPES; LIMA, 2019). Apesar dessa lacuna, existem estudos focados na fase de representação do conhecimento, que envolve abstrações e esquemas conceituais para representação de trilhas de aprendizagem, entre eles o trabalho de Schwab e outros (2017), o qual apresenta solução para navegação linear e hierárquica e navegação por meio de um mapa mental dentro de um mesmo curso.

As interfaces convergem escolhas advindas tanto da representação visual quanto do esquema de armazenamento dos dados. A integração dessas informações visa, sobretudo, atender as necessidades do usuário final. Nesse sentido, tanto o esquema conceitual quanto o esquema de armazenamento de dados favorecem a recuperação da informação. Com efeito, há presunção de que a existência de um modelo conceitual pode contribuir para o processo de organização e representação de trilhas de aprendizagem.

Assim, este estudo teve como propósito analisar interfaces de trilhas de aprendizagem, com vistas a extrair possíveis categorias e entidades a compor o modelo conceitual.

\section{METODOLOGIA}

Conforme exposto, este artigo traz resultados que buscam esboçar um metamodelo conceitual de representação de trilhas de aprendizagem, por meio da análise de interfaces de trilhas identificadas na revisão de literatura (LOPES; LIMA, 2019).

Para alcance desse objetivo, a análise de dados foi conduzida por meio das etapas sugeridas no método da análise de conteúdo, proposto por Bardin (2011). 
Em linhas gerais, Bardin (2011) recomenda que o processo de análise de conteúdo seja estruturado conforme as seguintes fases: 1) pré-análise, 2) exploração do material e 3) tratamento dos resultados, inferência e interpretação.

Na fase de pré-análise, exploraram-se as 12 interfaces identificadas durante a revisão de literatura, de modo a levantar possíveis entidades que pudessem compor o modelo conceitual. As interfaces identificadas representam apenas um extrato das interfaces de trilhas de aprendizagem existentes. Entretanto, como critérios de seleção dessas interfaces, optou-se por aquelas que estivessem disponíveis na web, sem necessidade de autorização prévia da instituição para exploração, além da menção explícita sobre adoção de trilhas de aprendizagem como estratégia de organização das soluções educacionais. Assim, a partir desses três critérios, foram selecionadas 12 instituições que dispunham de interfaces de organização e representação de trilhas de aprendizagem: Alison ${ }^{1}$, Besomebody ${ }^{2}$, Cbtnuggets $^{3}$, Cloudacademy ${ }^{4}$,

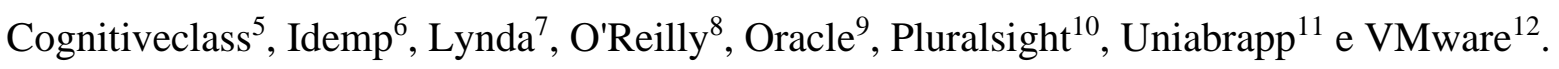

A cada análise, novas entidades eram identificadas de forma gradual. Após a finalização da análise inicial das 12 interfaces, o conjunto dessas entidades foi organizado em uma matriz.

$\mathrm{Na}$ fase de exploração do material, houve revisão e agrupamento dessas entidades em sete categorias de análise conforme a natureza, a saber: (1) esquemas de representação; (2) filtros e facetas; (3) tipos de soluções educacionais; (4) tipos de relacionamentos entre as soluções educacionais; (5) atributos das soluções educacionais; (6) processo de inscrição; e (7) procedimento de divulgação. A relação de categorias e as respectivas entidades não foram exaustivas, representando apenas elementos que puderam ser explicitamente identificados nas 12 interfaces analisadas.

A partir da revisão e do estabelecimento das categorias na matriz, na terceira fase da análise de conteúdo, nova verificação das interfaces foi realizada, no intento de verificar a

\footnotetext{
1 Disponível em: https://alison.com/learning-paths.

2 Disponível em: https://besomebody.com/paths/.

${ }^{3}$ Disponível em: https://www.cbtnuggets.com/learning-paths.

${ }^{4}$ Disponível em: https://cloudacademy.com/product/learning-paths/.

${ }^{5}$ Disponível em: https://cognitiveclass.ai/learn/all/.

${ }^{6}$ Disponível em: http://www.idemp-edu.com.br/IDEMP_MAPADOCONHECIMENTO_2015.pdf.

${ }^{7}$ Disponível em: https://www.lynda.com/learning-paths.

${ }^{8}$ Disponível em: https://learning.oreilly.com/learning-paths/.

${ }^{9}$ Disponível em: https://education.oracle.com/learning-paths/all_paths.

${ }^{10}$ Disponível em: https://www.pluralsight.com/product/paths.

${ }^{11}$ Disponível em:

http://www.uniabrapp.org.br/Cursos-Presenciais/Paginas/Trilhas-do-Conhecimento.aspx.

12 Disponível em: https://mylearn.vmware.com/mgrreg/plan.cfm?plan=70374\&ui=www_edu.
} 
existência ou não de cada uma das entidades nas interfaces selecionadas, cujos resultados são apresentados na seção seguinte.

\section{RESULTADOS}

Para a primeira categoria de análise, esquemas de representação, a maioria das interfaces possui a lista hierárquica como principal estrutura de organização. Além disso, mesmo nas duas interfaces que foi identificado o uso de mapa mental, ambas apresentam apenas links para as respectivas páginas das soluções educacionais, sem outras funcionalidades adjacentes (Gráfico 1).

Gráfico 1: Esquemas de representação

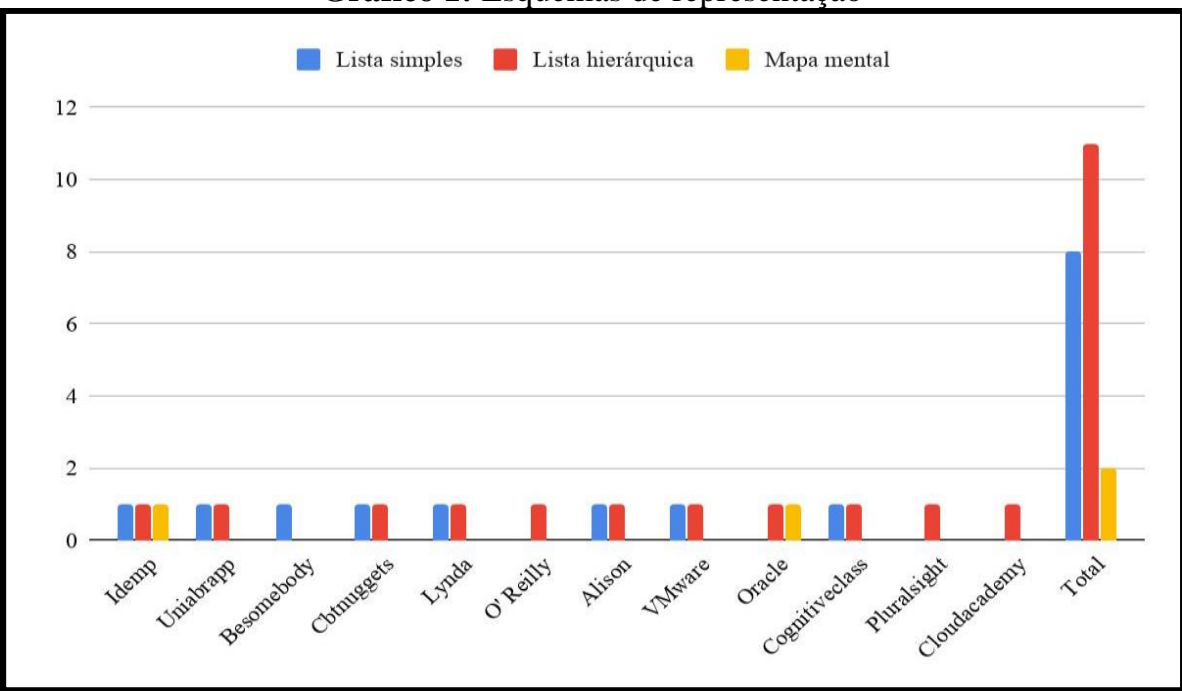

Fonte: elaborado pelas autoras (2020).

Na categoria (2) filtros e facetas, todas as interfaces possuem como filtro para realização de buscas tema, assunto, categoria ou área de conhecimento. Outro tipo de filtro que aparece na maioria das interfaces é a seleção por palavra-chave, o que reflete a existência de um modelo convencional de busca. Apesar de identificado em apenas duas interfaces, o filtro por data de início e término parece relevante no contexto das trilhas de aprendizagem, principalmente no âmbito da Educação Corporativa, cujo pressuposto é o de que o aluno tenha autonomia para realizar o planejamento do seu percurso de aprendizagem. Os filtros e facetas constatados para cada interface analisada estão expostos no Gráfico 2. 
Gráfico 2: Filtros e facetas

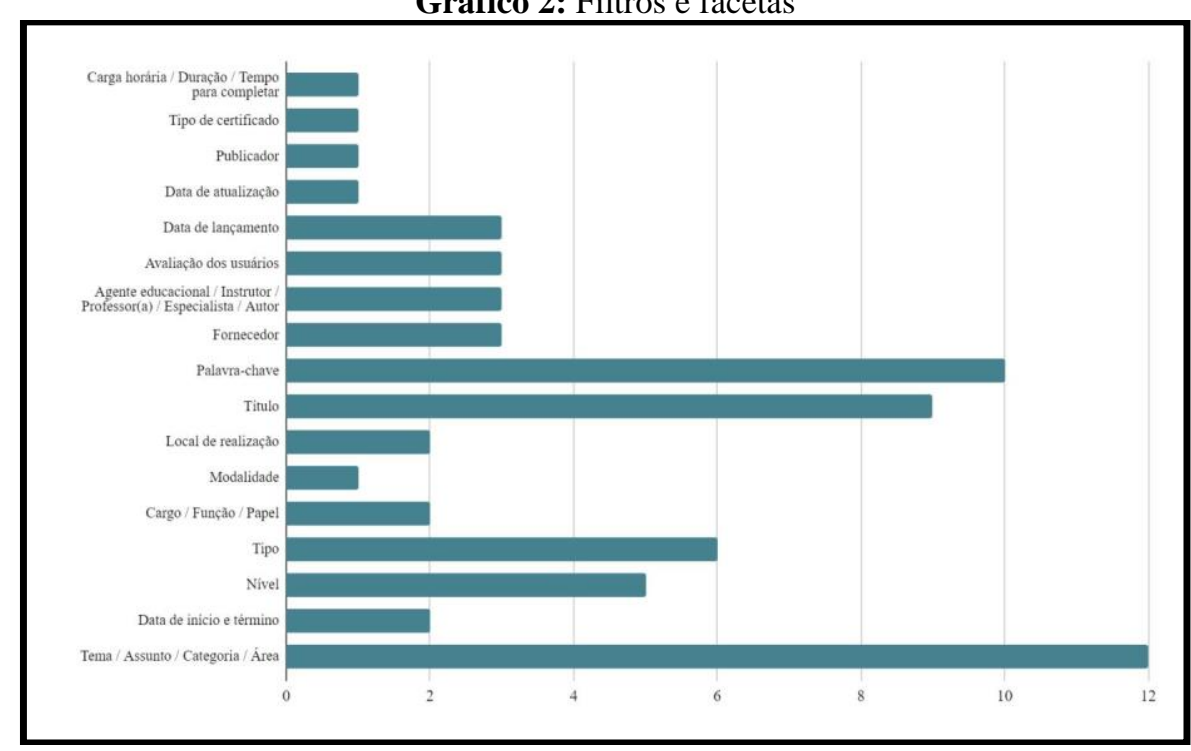

Fonte: elaborado pelas autoras (2020).

Em relação à categoria (3) tipos de soluções educacionais, predominam variados tipos de soluções educacionais; logo, infere-se, assim, a natureza multimodal das trilhas de aprendizagem. No entanto, a solução educacional do tipo curso é identificada em quase todas as interfaces, ao passo que outros tipos de soluções educacionais como estudo de caso e comunidade de prática aparecem em apenas uma das interfaces. Os resultados alcançados para essa categoria estão descritos no Gráfico 3.

Gráfico 3: Tipos de soluções educacionais

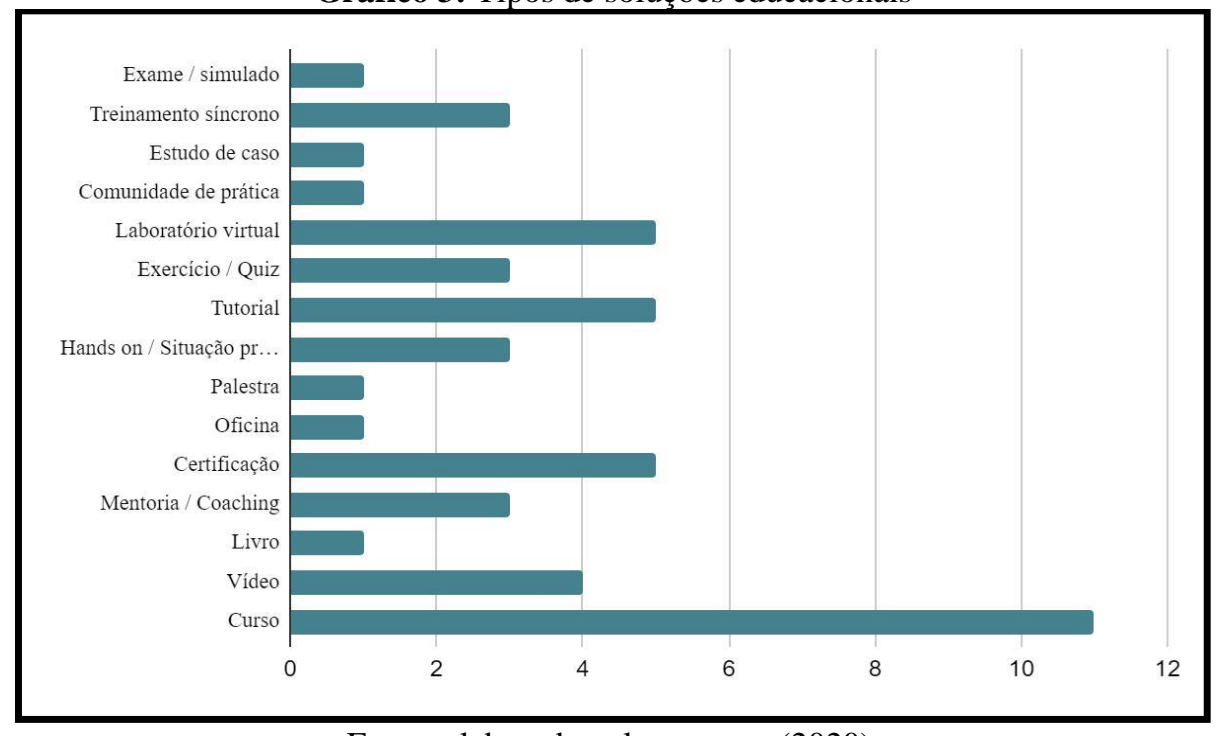

Fonte: elaborado pelas autoras (2020). 
Sobre a categoria (4) tipos de relacionamentos entre as soluções educacionais, os tipos de relacionamentos foram selecionados com base nos requisitos para elaboração e metodologias para modelos conceituais de hiperdocumentos propostos por Campos (2001). A partir dessa diretriz, os relacionamentos dos tipos categorial e hierárquico manifestaram-se em todas as interfaces, o que demonstra o esquema de organização das soluções educacionais. Os relacionamentos precedência e sucessão refletem uma relação do tipo partitiva e o entre categorias também pode ser identificado, o que confirma a relação entre soluções educacionais e outras entidades como nível, público-alvo, categoria temática, tipo, entre outras, haja vista que a relação de equivalência, mesmo estando restrita a variações terminológicas, não pôde ser visualizada em nenhuma das interfaces (Gráfico 4).

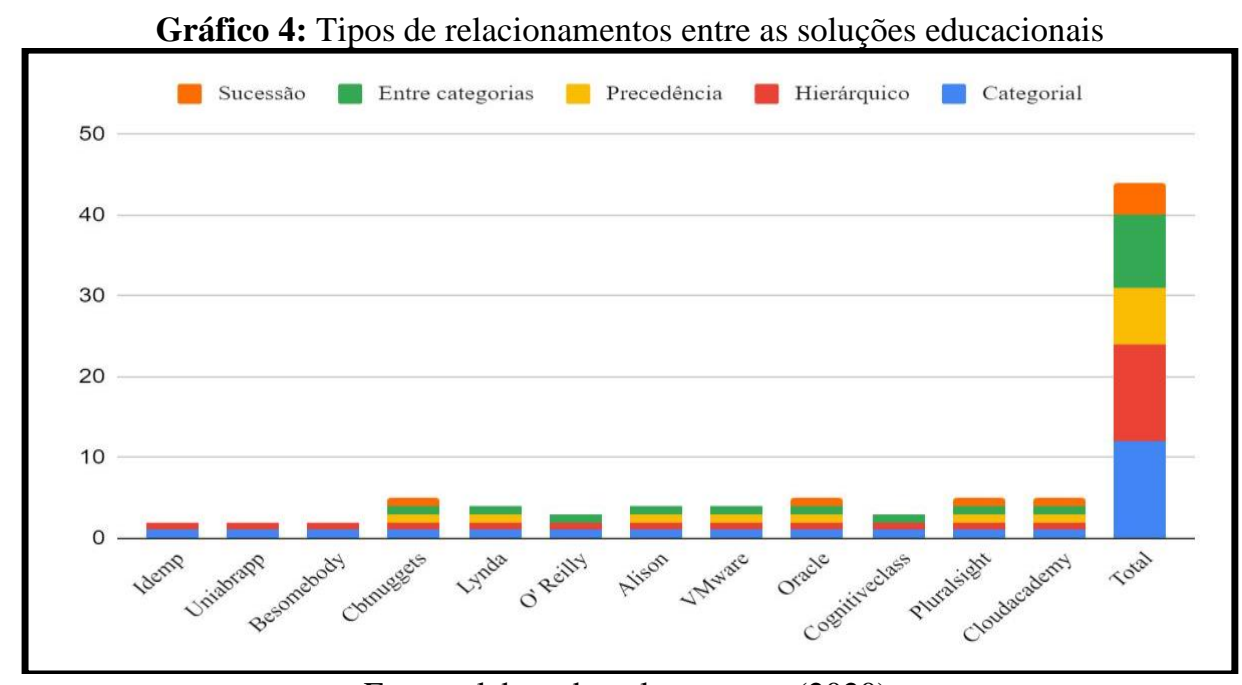

Fonte: elaborado pelas autoras (2020).

No que diz respeito à categoria (5) atributos das soluções educacionais, percebeu-se grande variedade de possíveis atributos, tendo em vista que na análise, a intenção foi identificar apenas os atributos que estavam explicitamente relacionados com a solução educacional. Muitos desses atributos apareceram organizados em fichas técnicas ou páginas de detalhamento de cada solução educacional (Gráfico 5). 
Gráfico 5: Atributos das soluções educacionais

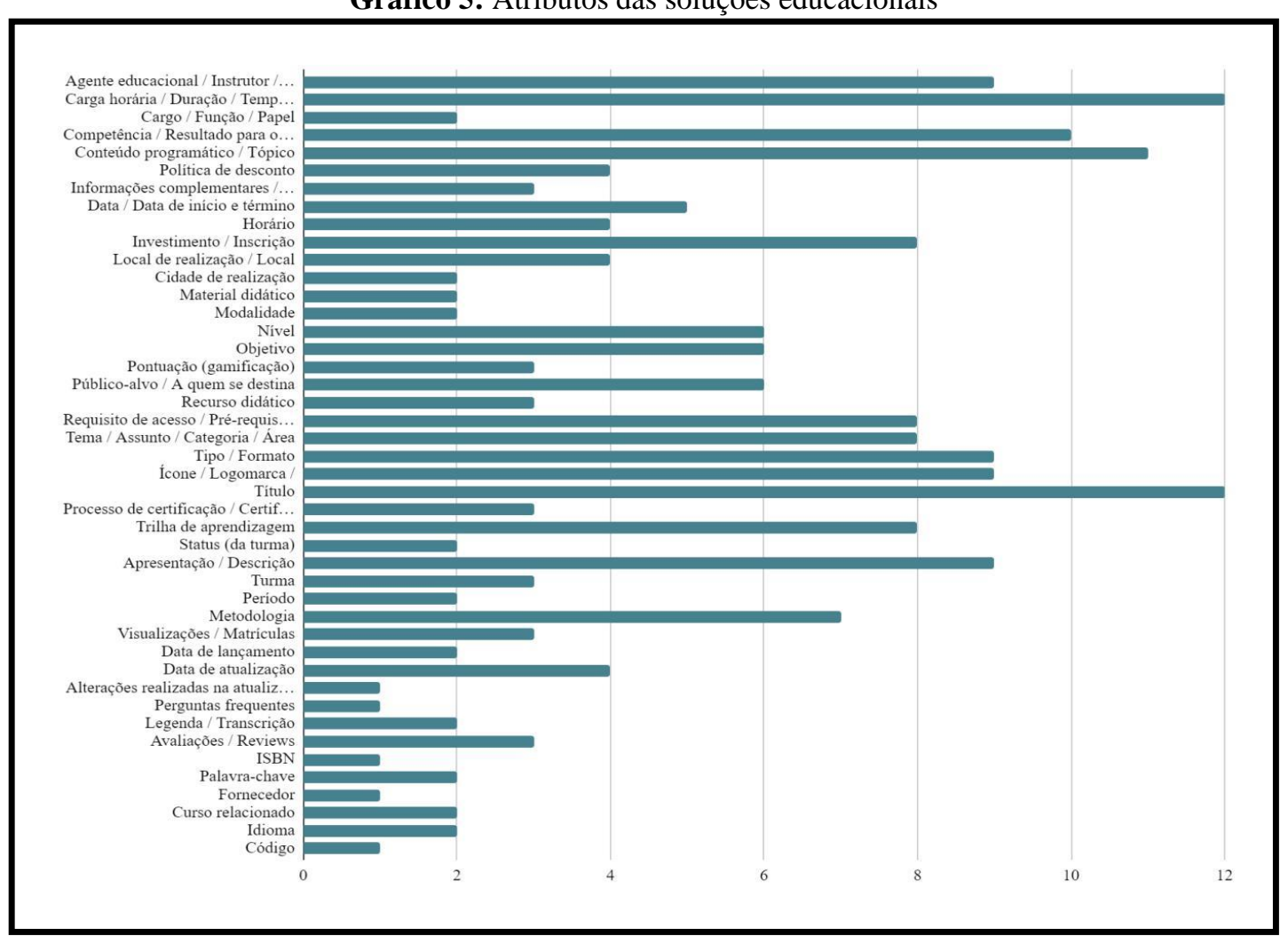

Fonte: elaborado pelas autoras (2020).

$\mathrm{Na}$ categoria (6) processo de inscrição, o foco centrou-se nas entidades que se relacionavam aos procedimentos ou orientações para matrícula ou inscrição. Destaca-se que a funcionalidade ser informado(a) sobre nova turma foi constatada em apenas três das interfaces, mesmo considerando o fato de terem sido localizadas soluções educacionais para as quais não haviam turmas disponíveis para inscrição. Da mesma forma, os requisitos técnicos e considerados mínimos para o computador não puderam ser identificados em muitas soluções educacionais, inclusive as que tratavam de temas que, pelo menos em tese, demandariam requisitos mínimos de hardware (Gráfico 6).

Gráfico 6: Processo de inscrição 


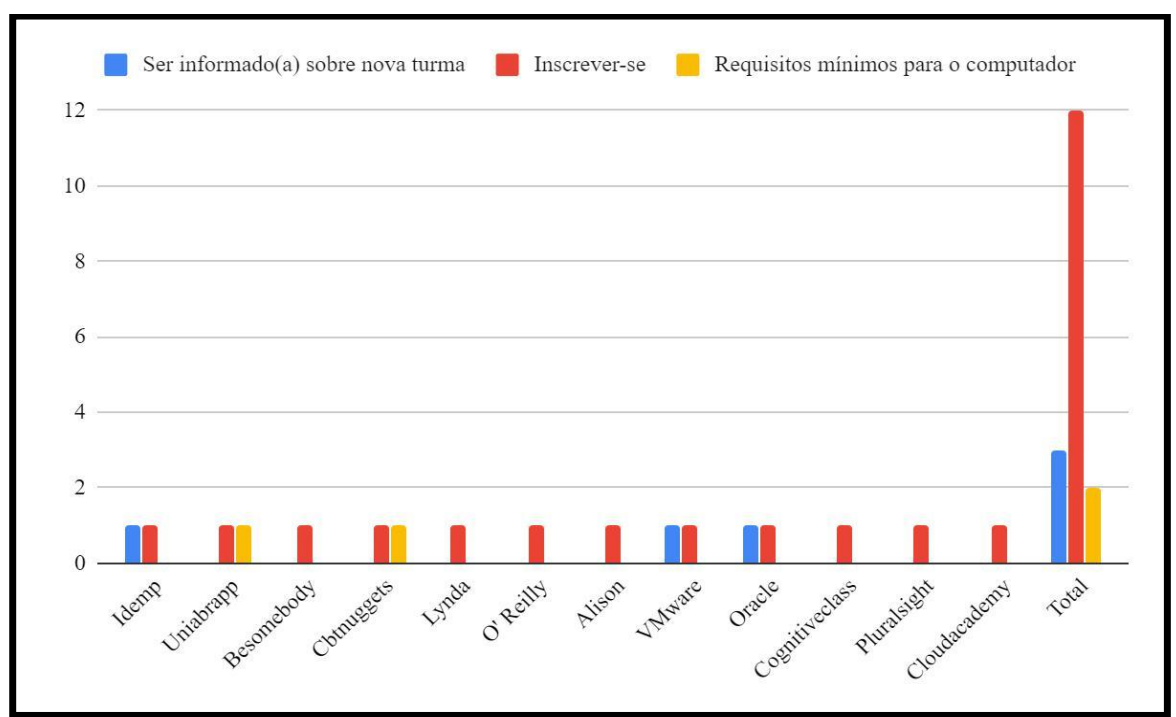

Fonte: elaborado pelas autoras (2020).

Não menos importante, na categoria (7) processo de divulgação, elementos de conexão com redes sociais foram constatados. Esses elementos permitem não apenas compartilhar informações de soluções educacionais, mas também, em algumas das interfaces, estabelecer conexões com outros alunos ou agentes educacionais. Os dados revelam, ainda, o uso de vídeos curtos como estratégia de apresentação das soluções educacionais, sendo um formato alternativo ao texto, que está presente na maioria das demais interfaces. O Gráfico 7 detalha esses elementos para cada instituição analisada.

Gráfico 7: Processo de divulgação 


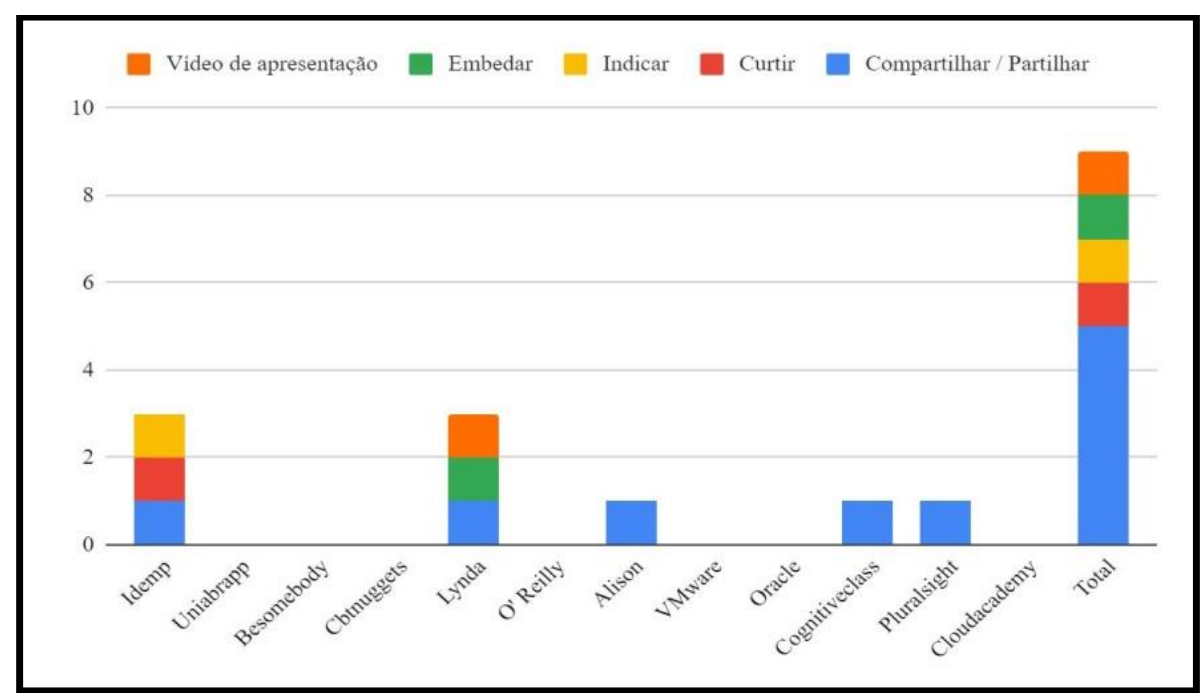

Fonte: elaborado pelas autoras (2020).

Portanto, a partir das considerações identificadas na literatura, constata-se a existência de diferentes elementos constitutivos de uma trilha de aprendizagem. Tais elementos ou categorias, além de caracterizarem esses recursos, também são representados e utilizados de diferentes maneiras, considerando a realidade e propósitos para o qual foram criados.

\section{ANÁLISE}

Retomando as categorias de análise adotadas neste estudo, em relação aos esquemas de representação, destaca-se que apesar de haver diversos estudos contemplando o uso de mapas mentais e mapas conceituais na organização de trilhas de aprendizagem como, por exemplo, em Velásquez e Ibañez (2015), Crossetti, Mesquida e Ibáñez (2012), Salinas, Benito e Darder (2011), Schwab e outros (2017) e Velásquez, Ibañez e Restrepo (2012), e, apenas duas das instituições apresentam esquemas de representação próximos a mapas mentais. Conforme destacado por Campos (2001), o modelo de representação convencional do hipertexto, baseado em uma representação de nós conectados por links, não é suficiente para representação de redes complexas. Nesse sentido, quanto maior o volume de soluções educacionais, tipos de relacionamentos entre essas soluções e também entre outras entidades, mais complexa essa rede poderá se apresentar. Dessa forma, mesmo havendo uma tendência de estudos para aplicação de mapas mentais e conceituais para representação visual de trilhas de aprendizagem, constatou-se a necessidade de mais pesquisas com relação a estratégias de visualização da informação, principalmente de redes complexas. 
Acerca dos filtros e facetas, a maioria das interfaces apresenta os filtros convencionais de busca como, por exemplo, nome ou palavra-chave. Resguardadas as diferenças entre os contextos, a busca facetada é uma funcionalidade atualmente presente em muitas interfaces de e-commerce. Nesse sentido, infere-se que a disponibilização de uma maior variedade de filtros no contexto de trilhas de aprendizagem tende a contribuir com a experiência do usuário. É importante frisar que filtros e facetas identificados representam categorias e entidades do modelo conceitual, e a escolha acerca de quais filtros ou facetas precisam compor a interface dependerá de diversas variáveis como: condições técnicas de implementação, preferências do público-alvo e leiaute da interface.

Com relação aos tipos de soluções educacionais, houve dificuldade para identificação das entidades, considerando diferentes terminologias adotadas. Mesmo com a intenção de analisar a descrição do tipo identificado, nem todas as interfaces dispunham dessa informação. Nesse sentido, é provável que essa dificuldade poderá se revelar também como empecilhos ao usuário final, visto não conseguir escolher a solução educacional adequada aos propósitos de aprendizagem. Destaca-se, assim, que o problema observado não se restringe à terminologia, mas também com relação ao uso de diferentes conceitos para se referir a uma mesma entidade.

Sobre a categoria tipos de relacionamentos entre as soluções educacionais, no âmago das interfaces das trilhas de aprendizagem, os dados esclarecem que os relacionamentos dos tipos precedência e sucessão são importantes para estruturação dos percursos formativos dentro das trilhas, o que possibilita, portanto, a identificação não apenas do requisito de acesso à determinada solução educacional (predecessor), mas também das próximas soluções educacionais indicadas para a sequência do percurso formativo (sucessor). Em algumas interfaces, há indicação de outras soluções educacionais como predecessores e sucessores, e não apenas textos não estruturados indicando requisitos de acesso ou soluções educacionais complementares. Também foi observado o uso da funcionalidade de recomendação, comum em serviços de $e$-commerce, que se baseia na inserção prévia de parâmetros, por exemplo, assuntos de interesse do usuário ou mesmo avaliações diagnósticas para detectar o nível de conhecimento do usuário em determinado assunto.

As análises indicaram problemas de natureza terminológica e conceitual em relação aos atributos das soluções educacionais, como também no que se refere à categoria tipos de soluções educacionais, o que também dificultou a identificação das entidades e posterior classificação. Apesar de haver grande variedade de entidades, nem todos os atributos 
registrados devem estar necessariamente visíveis para o usuário final como, por exemplo, identificadores e códigos, essenciais para o processo de organização e recuperação da informação, mas de menor relevância para compor a interface. É necessário, nesse contexto, avaliar quais atributos vão compor o modelo conceitual, quais deles farão parte do modelo do banco de dados e quais serão exibidos para os usuários finais. A análise às variáveis já mencionadas com relação à escolha de possíveis filtros e facetas demonstra, também, o problema do transbordamento cognitivo (CONKLIN, 1987; LIMA, 2004, 2015). Assim, caso o usuário não tenha condições de processar o volume de informações exibido na tela, poderá haver perda de informações importantes para o percurso formativo do aprendiz. Nesse sentido, é recomendado proceder à avaliação sobre quais informações são essenciais e como exibir essas informações de forma a contribuir para a experiência do usuário.

No que tange aos processos de inscrição e divulgação, esses processos estão focados muito mais nas soluções educacionais e não exatamente nas trilhas de aprendizagem. Algumas instituições adotam a inscrição por trilha de aprendizagem, disponibilizando, inclusive, a página específica para que o usuário possa acompanhar o percurso formativo dentro da trilha como um todo e não apenas limitado à solução educacional. Os dados analisados indicam o uso de funcionalidades que permitem a conexão com redes sociais. Entretanto, na maioria das interfaces, o propósito desses links restringe-se à divulgação e ao compartilhamento de informações e não ao estabelecimento de conexões entre usuários finais ou entre esses e os agentes educacionais.

\section{CONSIDERAÇÕES FINAIS}

Este estudo apresentou alguns resultados de uma pesquisa de mestrado realizada no âmbito do Programa de Pós-Graduação em Gestão e Organização do Conhecimento da Escola de Ciência da Informação, Universidade Federal de Minas Gerais, Brasil. O objetivo delimitado para este artigo foi esboçar um metamodelo conceitual de representação das trilhas de aprendizagem. Com o intuito de criar esse metamodelo, foram analisadas interfaces de trilhas identificadas na revisão de literatura. A partir dessa análise, foi possível identificar uma variedade de categorias e entidades que foram usadas para a composição do modelo conceitual para organização e representação de trilhas de aprendizagem. 
Mesmo em face da necessidade de refinamento dos elementos identificados, principalmente do ponto de vista de tratamento terminológico, considerou-se que o levantamento realizado contemplou necessidades reais de usuários finais. Logo, esse resultado evidenciou o pressuposto de que tais necessidades foram consideradas no momento de criação das respectivas interfaces. De qualquer modo, avalia-se como necessário o refinamento do modelo conceitual proposto, considerando, principalmente, a possibilidade de uso de um vocabulário controlado, visando o enriquecimento semântico.

\section{REFERÊNCIAS}

ADESINA, Ayodeji; MOLLOY, Derek. A Business Process Management based Virtual Learning Environment-Customised Learning Paths. In: INTERNATIONAL CONFERENCE ON COMPUTER SUPPORTED EDUCATION, 3., 2011, [s. l.]. Anais [...]. [s. l.: s. n.], 2011. p. 365-368.

BALASUBRAMANIAN, V. et al. State of the art review on hypermedia issues and applications. New Jersey: Graduate School of management, 1994. Disponível em: http://csis.pace.edu/ marchese/CS835/Lec3/balasubramanian94.pdf. Acesso em: 20 mar. 2020.

BARDIN, Laurence. Análise de conteúdo. 4. ed. Lisboa: Edições, 2011.

CAMPOS, Maria Luiza de Almeida. A organização de unidades do conhecimento em hiperdocumentos: o modelo conceitual como um espaço comunicacional para realização da autoria. 2001. Tese (Doutorado em Ciência da Informação) - Instituto Brasileiro de Informação em Ciência e Tecnologia, Universidade Federal do Rio de Janeiro, Rio de Janeiro, 2001.

CAMPOS, Maria Luiza de Almeida. Modelização de domínios de conhecimento: uma investigação de princípios fundamentais. Ciência da Informação, Brasília, v. 33, n. 1, p. 2232, jan./jun. 2004. Disponível em: http://www.scielo.br/scielo.php?pid=S0100$19652004000100003 \&$ script=sci_abstract\&tlng=pt. Acesso em: 20 mar. 2020.

CONKLIN, Jeff. Hypertext: An Introduction and SurvevJ. IEEE computer, v. 20, n. 9, p. 1741, 1987.

CROSSETTI, Bárbara de Benito; MESQUIDA, Antònia Darder; IBÁÑEZ, Jesús Salinas. Los itinerarios de aprendizaje mediante mapas conceptuales como recurso para la representación del conocimiento. Revista Electrónica de Tecnología Educativa, n. 39, p. 1-14, mar. 2012.

FREITAS, Isa Aparecida; BRANDÃO, Hugo Pena. Trilhas de Aprendizagem como Estratégia para Desenvolvimento de Competências. In: ENCONTRO DA ASSOCIAÇÃO DOS PROGRAMAS DE PÓS-GRADUAÇÃO EM ADMINISTRAÇÃO, 29., 2005, Brasília. 
Anais [...]. Rio de Janeiro: Anpad, 2005. Disponível em: http://www.anpad.org.br/admin/pdf/enanpad2005-gpra-0316.pdf. Acesso em: 20 mar. 2020.

LEGGETT, John; SCHNASE, John; KACMAR, Charles. A Short Course on Hypertext. 1989. Disponível em:

https://pdfs.semanticscholar.org/7c51/fb83d29ad6bdd05340324873d92969c786d6.pdf. Acesso em: 20 mar. 2020.

LIMA, Gercina Ângela Borém. Mapa hipertextual (MHTX): um modelo para organização hipertextual de documentos. 2004. Tese (Doutorado em Ciência da Informação) - Escola Ciência da Informação, Universidade Federal de Minas Gerais, Belo Horizonte, 2004.

LIMA, Gercina Ângela de. MHTX: Modelagem hipertextual para organização de documentos: princípios e aplicação. Rio de Janeiro: Interciência, 2015.

LIMA, Gercina Ângela de. Organização da informação para sistemas de hipertextos. In: NAVES, Madalena Martins Lopes; KURAMOTO, Hélio (org.). Organização da informação: princípios e tendências. Brasília: Briquet de Lemos/Livros, 2006. p. 99-116.

LOPES, Patrícia; LIMA, Gercina Angela. Estratégias de Organização, Representação e Gestão de Trilhas de Aprendizagem: uma revisão sistemática de literatura. Perspectivas em Ciência da Informação, Belo Horizonte, v. 24, n. 2, p. 165-195, 2019. Disponível em: http://www.scielo.br/scielo.php?script=sci_arttext\&pid=S1413-99362019000200165. Acesso em: 20 mar. 2020.

LOPES, Patrícia. Proposta de metodologia para organização e representação de trilhas de aprendizagem no contexto da educação corporativa na administração pública. 2018. Dissertação (Programa de Pós-Graduação em Gestão e Organização do Conhecimento) Escola de Ciência da Informação, Universidade Federal de Minas Gerais, Belo Horizonte, 2018. Disponível em: https://repositorio.ufmg.br/handle/1843/BUOS-BD9P6W. Acesso em: 16 mar. 2020.

LUBCHAK, Vladimir; KUPENKO, Olena; KUZIKOV, Borys. Approach to dynamic assembling of individualized learning paths. Informatics in Education, v. 11, n. 2, p. 213$225,2012$.

NELSON, Ted. A File Structure for the Complex. National Conference, 1965. Disponível em: http://csis.pace.edu/ marchese/CS835/Lec3/nelson.pdf. Acesso em: 24 ago. 2018

PEREZ, Celso Roberto. A modelagem de documentos estruturados multitmídia integrando sistemas de hipertextos e ODA/ODIF. 1994. Disponível em: http://www.lume.ufrgs.br/handle/10183/25461. Acesso em: 15 mar. 2020.

RADA, Roy. Trends in: A critical review. Informmion Processing \& Monogemenf, v. 27, n. 6, p. 659-617, 1991.

SALINAS, Jesús; BENITO, Barbara de; DARDER, Antonia. Los mapas conceptuales como organizadores del proceso de enseñanza-aprendizaje: los itinerarios de aprendizaje. 
Investigació I Innovació Educativa I Socioeducativa, v. 3, n. 1, p. 63-74, 2011.

SCHWAB, Michail et al. Booc. io: An Education System with Hierarchical Concept Maps and Dynamic Non-linear Learning Plans. IEEE Transactions on Visualization and Computer Graphics, v. 23, n. 1, p. 571-580, 2017. Disponível em:

https://www.ncbi.nlm.nih.gov/pubmed/27875172. Acesso em: 15 mar. 2020.

GÁMEZ SUAZO, Irma; GARITA RODRÍGUEZ, César; CHACÓN RIVAS, Mario. Generating adaptive learning paths in e-learning environments. In: CONFERÊNCIA LATINOAMERICANA EN INFORMÁTICA, 38., 2012, [s. l.]. Anais [...]. [S. l.: s. n.], 2012. p. 1-10. Disponível em: https://ieeexplore.ieee.org/document/6427219. Acesso em: 16 mar. 2020 .

VELÁSQUEZ, Olga Lucía Agudelo; IBAÑEZ, Jesús Salinas; RESTREPO, Claudia Zea. Desarrollo de competencias apoyado en itinerarios de aprendizaje flexibles basados en mapas conceptuales. In: INTERNATIONAL CONFERENCE OF CONCEPT MAPPING, 5., 2012. Valletta, Malta. Proceedings [...]. Valletta: IHMC; University of Malta, 2012. v. 1, p. 1561632. Disponível em: http://eprint.ihmc.us/233/. Acesso em: 17 mar. 2020.

VELÁSQUEZ, Olga Lucía Agudelo; IBAÑEZ, Jesús Salinas. Itinerarios de aprendizaje flexibles basados en mapas conceptuales. Journal of New Approaches in Educational Research, v. 4, n. 2, p. 70-76, 2015. Disponível em: https://www.learntechlib.org/p/151681/. Acesso em: 20 mar. 2020.

YANG, Fan. Learning Path Construction in e-Learning-What to Learn and How to Learn? 2012. Thesis (Doctorate in Philosophy) - Durham University, Durham, 2012. Disponível em: http://etheses.dur.ac.uk/3359/. Acesso em: 18 mar. 2020. 